\title{
Supplying of Products (Herbs) on the EU Market, Imereti Agrozone, Export Opportunities
}

\author{
Shota Lominashvili ${ }^{1}$, Naira Virsaladze ${ }^{2}$ and Irine Tchanturidze ${ }^{3}$
}

\begin{abstract}
Imereti is located in the historical Colchis valley. With its teritory and potential of resourses it is one of the biggest regions in Georgia. The population of the nearby villages of the Rioni River in Imereti region have been following the cultivation of different crops. In the recent past, the greenhouse herbs production is remarkable. The products are distinguished by ecological cleanness and high quality. Within the DCFTA (Deep and Comprehensive Free Trade Area) and SME (Small and medium-sized enterprises ) projects it is important to ensure opportunity of certain products constant delivery to european countries. We have investigated this issue for this purpose. An anonimous research of ordinary people and detailed survey of entrepreneurs involved in exports, about land and other resources, financial issues and rediness of sociaty to start business,was carried out to reveal various problems in this field. Interesting results were received after making statistical analysis and relevant conclusions were made. Specifically, research finds that there are material and labor resources and sustainable climatic conditions for the continuous supply of herbs on the EU market in the Imereti Agrozone. In addition, the creation of green and greenhouse industries requires interested population to provide targeted state support for credit availability, quality seed materials and pest control and irrigation infrastructure. The vital direction is to find sustainable export markets and implement projects supporting the creation of agricultural cooperatives.
\end{abstract}

Keywords: European integration, Export, Imereti agrozone, Financial material and labor resources, Continuous delivery, Standing market

The purpose of this survey is to study the potential and all the resources of ImeretyAgrozone to supply EU countries continuously with enough amounts of Georgian production herbs according to DCFTA and the prime minister's announcement

For the purpose of research, two different content questionnaires were held: first anonymous random survey and the second, detailed questionnaires of the herb producers.

After the random anonymous surveys of the population of target area the following issues have been revealed:

1. 118 respondents, $66 \%$ of inquired people have information about DCFTA and SME.

2. Only 70, less than 39\% of respondents have greenhouse farms from enquirred 180 respondents

3. 53 respondents $(76 \%)$ from those 70 who already have greenhouse farming have desire to expand and from those 110 respondents who don't have greenhouse farming yet 60 respondents $(55 \%)$ are willing to create it.

\footnotetext{
1Doctor of Economics, Associated Professor, Head of Economic Department in Akaki Tsereteli State University,Kutaisi,Georgia

${ }^{2}$ Academic Doctor of Economics, Associate Professor of Economics Department of Kutaisi, Akaki Tsereteli State University, Head of Statistics Department

${ }^{3} \mathrm{PhD}$ student of Economics at Akaki Tsereteli State University, Kutaisi, Georgia
} 
17 respondents have vegetable gardens. 44 respondents don't have desire to set up greenhouse farming, but 7 from these people still produce herbs in their vegetable garden. It is obvious that the population is interested in creating new farms and the expansion of existing greenhouse farms.

4. a. 83 ( or $46 \%$ ) out of 180 respondents produce herbs in the vegetable gardens without greenhouse farming.

a.a 31 respondents $(37.3 \%)$ annually sell roughly 1 tone herb.41 of them sell nearly 1-3 tones and more than 3 tones sell 9 respondents.

a.b. 31 respondents out of 81 consider producing more than 1 tone herbs possible.

41 respondents from 1 to 3 tones and 11 of them think about producing more than 3 tones .

a.c. 23 out of 83 respondents are willing to produce 1 ton of greens, From 1 to 3 tons 46 respondents, more than 3 tones - 14 respondents. It is intended to increase the production of greens with the field method.

b. The rest from 97 respondents who don't grow herb in the garden fields name the following interrupting factors: 15 respondents believe that this method of making herbs is not profitable because of the small income and barrenness of the land. The absence of trading marketis the reason for 4 respondents, 4 respondent say because of the lack of land, 14 of them talk about financial problems, absence of irrigation system and water shortage - 7 respondents; 13- lack of time, 9 respondents refrained from responding.Age and physical condition was named by 17 respondents; It is noteworthy that only 4 respondents name the key market as an interrupting factor in the producing herbs in vegetable gardens. In our opinion it is due to the fact that in vegetable gardens herb is produced in spring and summer when it is sold without problems because of high demands in local markets and tourism season. In contrast, the different problems appear in the greenhouse producing of herbs in late Autumn

5. The absolute majority of interviewed respondents (156 respondents) consider the capital greenhouse (metal frame with glass) as efficient, the metal frame with cellophane is thought to be effective just by 24 respondents, the wooden frame does not seem to be effective in any way, though in reality the majority of greenhouses are exactly wooden frames with cellophane on it.

6. because of current prices on herbs and energy sources (gas, electricity) 117 respondents $(61 \%)$ consider capital greenhouses an profitable ones and the rest $39 \%$ think the opposite.

7. When asked what the type of annual agricultural products do you consider to be profitable except of herbs, the interviewed 180 respondents did not name alternative culture therefore, 40 respondents refrained from answering. From the rest of the 140 respondents 90 think that cucumber production is profitable; 100-planting the tomatoes; Onions - 32 garlic and Bulgarian pepper separately-9; Salad 10 Strawberry and raspberry16; Potato-4; Maize-5; Production of soy-bean, earthnut, melon, watermelon and other agricultural crops - 7. It can be concluded that entrepreneurs consider growing tomatoes (100 respondents) and cucumbers (90 respondents) the most popular after herbs.

8. 137 respondents $76 \%$ from enquired ones have no information about ImeretiAgrozone. 
9. 128 respondents $71 \%$ don't know The Law on Agricultural Cooperatives, accordingly they are not cooperative members. When we talk about the recognition of the law, we take into consideration not knowing its content but the information about its existence and a result like this clearly indicates the low efficiency of work and the deficit of the implemented activities on this issue

10. Only 4 people out of 180 inquired ones are members of the cooperative, that proves our conclusion about the result of previous (9) question.

11. Despite the fact that they do not have information about cooperative in case offering 31 respondents that is only $17 \%$ have desire to become its members, this strengthens the conclusions made in previous questions and points to ineffective activities of local self-governments and relevant structures.

12. 125 respondents $-69,4 \%$ of inquired people have no idea about cooperative prospects at all. In case of guarantee of legislative base and financial support on products of sales Cooperative membership is acceptable and positively evaluated by 37 respondents, or $20.5 \%$ of respondents. 36 respondents have a negative attitude towards cooperative membership, that is $20 \%$. The results obtained from the survey have further reinforced the conclusion, about cooperatives, and the lack of working activities with the population.

13. 25 respondents did not make a summary comment on the poll. 49 respondents or $27.2 \%$ out of 155 residents consider necessity of activating state assistance for the development of agriculture. 65 respondents, or 36\%, think that the trading market and low price is a problem. 20 respondents $(11 \%)$ talk about providing chemicals and their control. Creation of the anti-flooding system is necessary for 1 respondent and 20 respondents $(11 \%)$ are willing to create an irrigation system. 19 respondents $(10,5 \%)$ talk about lack of financial resources and are willing to get cheap agro loans.

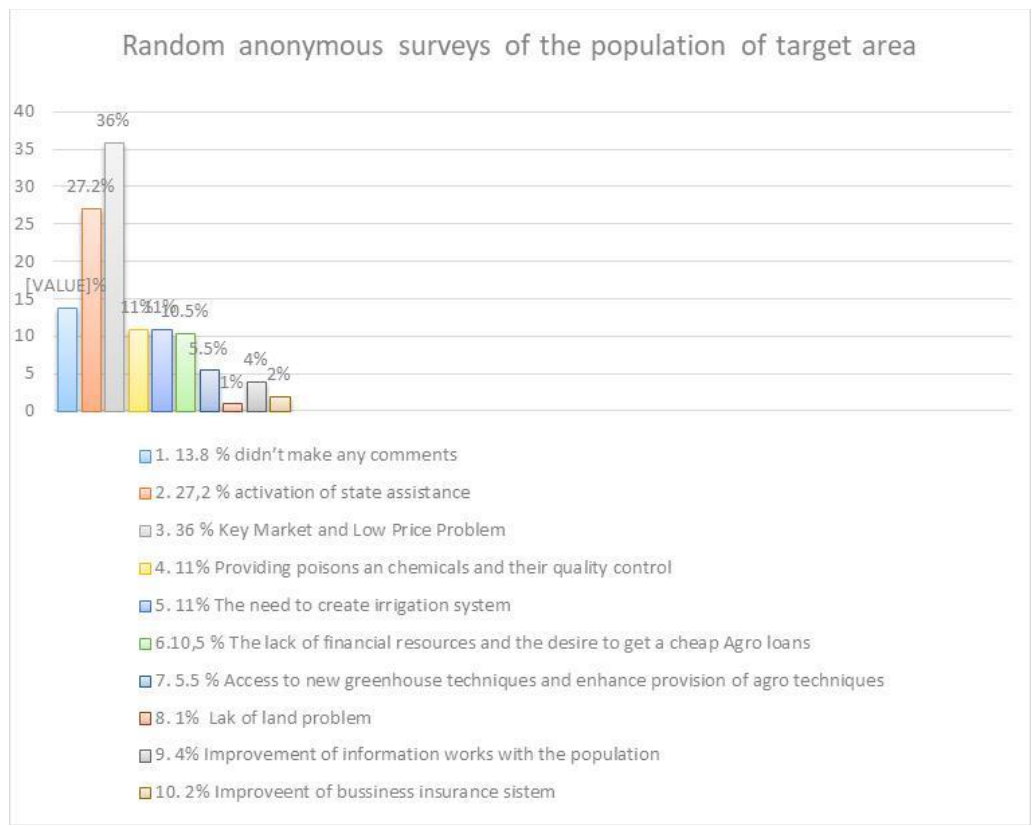

(C) 2019 The Authors. Journal Compilation ～(C) 2019 European Center of Sustainable Development. 
10 respondents $(5,5 \%)$ require to have access to new greenhouse technologies and improve their condition with agrotechnics. Two respondents who have the desire to create cooperatives have a problem of land; 8 respondents name the imminence of improving the information work with the population. Five respondents, in order to compensate the damage inflicted by natural phenomena, sees necessitates of business insurance regulations and etc.

\section{Compound results and conclusions of detailed inspection of entrepreneurs engaged in herbs production and export}

1. $23(85.2 \%)$ out of 27 enquired entepreneurs have information about DCFTA and SME

2. 17 out of 27 surveyed (or 63\%) think that there are appropriate standard resources to supply EU countries continuously with sufficient amount of herbs (coriander, parsley, fennel)

3. 24 out of 27 respondents surveyed are engaged only in the production of greens; 2 Purchase and export of greens, and one is engaged in producing herbs as well as its exports.

4. 14 out of 27 respondents surveyed (52\%) are mainly engaged in production of coriander, parsley and fennel. Only fennel produces - 12 (44\%); Parsley and Coriander 9 (33\%); Only parsley - 2 (7\%). Together with these plants, entrepreneurs also produce onions, lettuce, peppers, but in a much less amount. So, in these villages, the production of fennel, parsley and coriander is the leading, fennel in an advantage

5.15 out of 27 respondents surveyed (ie 56\%) mainly sell products in the local market, while the rest of the products is exported in Russia and Ukraine. Entrepreneurs name Russia and Ukraine as the main exporting countries

6. The most demanded herbs in Ukraine and Russia are fennel and partially parsley.

7. 13 out of 27 surveyed entrepreneurs have a metal carcass greenhouse with cellophane, 10 have wooden carcass with cellophane and 3 have both kind of greenhouses. Only one entrepreneur uses capital greenhouse (metal carcass with glass)

8. The majority of enquired entrepreneurs consider capital greenhouse as efficient, just 2 entrepreneur prefer greenhouse with metal carcass and cellophane and only one thinks wooden greenhouse with cellophane covering is better. We think that these answers are due to the low cost of such kind greenhouses.

9. As for greenhouse technique, it is available for 12 entrepreneurs and is satisfactorily evaluated,4 entrepreneurs have their own tractor but partially manually cultivate the land.While the rest 11 entrepreneurs (41\%) are dissatisfied with the lack of government assistance in this regard.

10. All surveyed entrepreneurs use specially featured seeds

11. It should be noted that most of them do not know and $52 \%$ of surveyed entrepreneurs and do not use appropriate literature in the field of producing herbs.

12. Also, 55.5\% of interviewed entrepreneurs do not have a written knowledge of the means of crop protection.

13. The majority of respondents $(82 \%)$ report that the qualified agricultural staff (agronomists) are not enough to provide qualified counseling when needed.

14. When asked how much is $1 \mathrm{~kg}$ herb. The value of the greens in $93 \%$ of the entrepreneurs' opinions are almost similar and varies from 1 to 3 GEL, namely, 
coriander from 0,8 to 2GEL; Parsley from 0.8 to 2 GEL; While the fennel is between 1 to 3 GEL. The cost of producing one KG herb for one enterpreneur is from 1 to $4 \mathrm{Gel}$, Coriander production costs $8 \mathrm{GEL}, 1 \mathrm{~kg}$. Parsley and fennel production is 5 GEL.

15. As it seems from the poll entrepreneurs' opinion about the price of greens is quite differentiated. For the purpose of generalizing the issue and the generalization of the indicator we used a weighted medium, which resulted in a weighted average price of $1 \mathrm{~kg}$ of greens (parsley, coriander and fennel) and equal to 4,5 GEL.

16. As the respondents say, the price of herbs is quite variable according to the season: the price of the coriander varies between 0,8 to 15 GEL, parsley from 1 to 12 GEL, and fennel also from 1 to 5 GEL.

17. $17(63 \%)$ enterpreneurs are aware of standards required from EU when exporting their products. 12 of them are directly busy doing this activity.

18. All entrepreneurs report that there is no quality laboratory for checking the quality of products of agriculture in their area.

19. None of the surveyed entrepreneurs have a warehouse for the production of greens. 20. When asked whether the management of the region and the local self-governments of the municipality helps the entrepreneurs in solving the problems in their activities or not, 25 from 27 entrepreneurs, or $92.5 \%$, think that they do not care about their activities and that they do not have any support from the regions self-government leaders. While 2 entrepreneurs believe that such assistance partially exists.

\section{Detailed questionnaires of the herb producers}

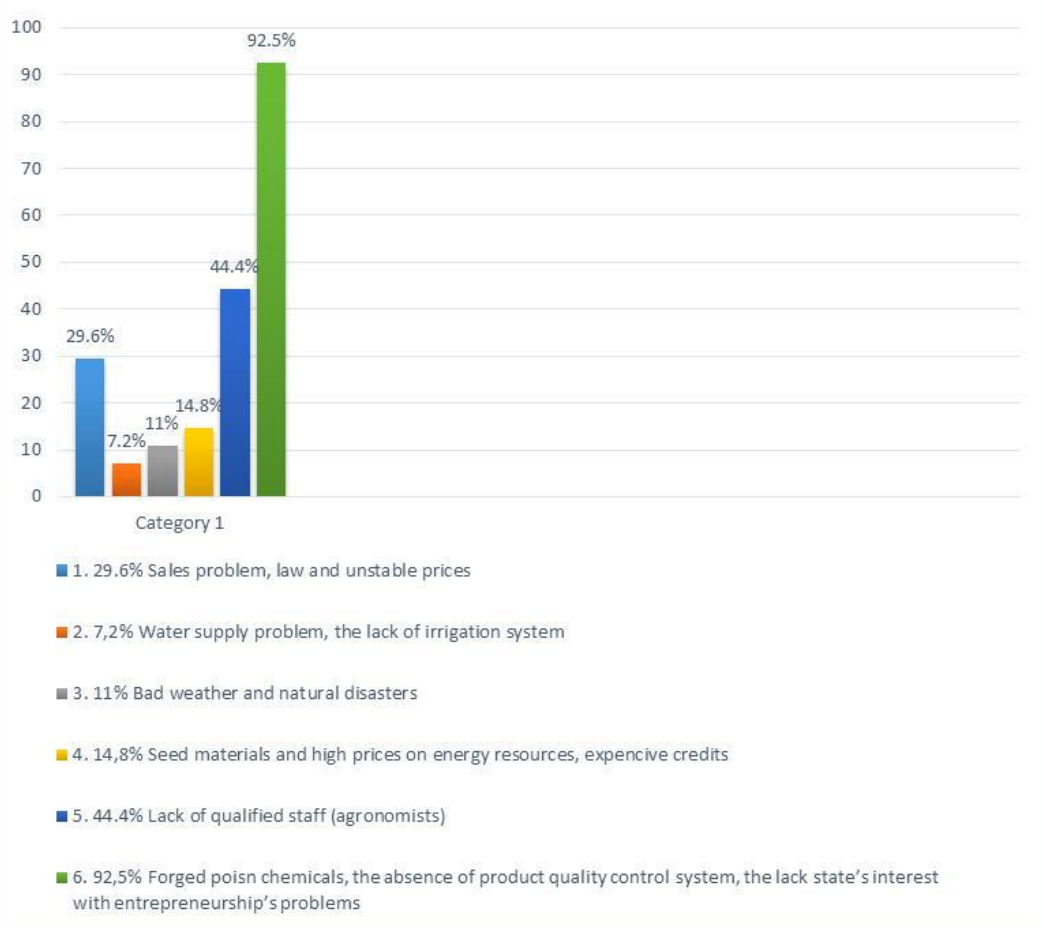


21. The inquired entrepreneurs face certain difficulties in producing and selling the product:

a. The problem of selling, more or less low and unstable prices - 8 entrepreneurs, ie $29,6 \%$ of respondents;

b. Water problem and, consequently, the absence of irrigation system - 2 entrepreneurs, ie $7.2 \%$ of respondents;

c. Bad weather and natural disaster - 3 entrepreneurs, ie $11 \%$;

d. High prices on seed material and energy resources, the high rate of credits - 4 entrepreneurs, ie $14.8 \%$;

e. Lack of qualified personnel - 12 entrepreneurs, ie 44.4\%;

f. Fraudulent poison-chemicals, lack of product quality control system, government's disinterestedness towards the entrepreneurs and etc. -25 entrepreneurs, or $92.5 \%$ of respondents.

\section{Conclusions}

Based on the statistical processing of the information obtained in the research process and the opinion of the respondents, a clear picture was revealed and relevant conclusions were made. Namely:

1. The majority of respondents $(75 \% 5 \%)$ have information about DCFTA and SME

2. The results of the study show that in the ImeretiAgrozone area actually exist material (land), labor resources and sustainable climatic conditions for the continuous supply of herbs to the EU market.

3. The main herb culture is reviled in the surveyed population: fennel, parsley and coriander, the fennel favors

4. A significant portion of the population (more than half) sells greens mainly on the local market, while some of them export products in Russia and Ukraine. Entrepreneurs name Russia and Ukraine as the main exporting country, where the most demanded herbs are fennel and partially parsley.

5. The absolute majority of interviewed respondents think that the capital greenhouse (Metal Frame with glass) is effective. The metal frame with cellophane is considered as effective for a small fraction of respondents, the greenhouse with wooden frame and cellophane is not considered as effect by any respondents, while most greenhouses are actually like this.

6. According to the results of the survey, the opinion of entrepreneurs on the minimum price of greens varies greatly. However, their common sense is that the price on the greens should be much higher, since insome seasons, the money received from realization neither can get profit, nor even pay the expenses.

7. The sharp change in greens prices is mainly based on seasons. The highest price is recorded in winter when the temperature is very low. Prices also grow sharply in the summer, especially in hot weather and droughts.

8. It should be noted that such change in green prices does not affect its stable production because the demand for greens is inflexible because it represents a substantial ingredient in different types of production and changes the final value of products only slightly. As studies have shown, the import of greens in the EU was growing during the 
economic crisis, and without the reduction of prices on products.

9. Most of the respondents do not use relevant literature and have little knowledge from the books in this field.In their opinion, qualified agricultural staff (agronomists) are not sufficiently qualified to give accurate advice when necessary

10. Only $2 \%$ of respondents are members of cooperative. In case of guarantee of regulation, financial support and sales legislative base, membership of cooperative is acceptable and positively evaluated by $20.5 \%$ of respondents. The results further reinforce the conclusion about cooperatives, the lack of works with the population about this issue.

11. People who produce herbs and are interested in creating expanding greenhouse farms are in need of State support for credit availability and flexible insurance system, quality seed materials, efficient ways to combat pests and develop irrigation infrastructure.

12. The vital direction is to find a sustainable export market - implementing projects that support development of agricultural cooperatives. 